\title{
Crossmodal transfer of conceptual responding in children
}

\author{
DONALD J. TYRRELL*† \\ Franklin and Marshall College, Lancaster, Pennsylvania 17604
}

\begin{abstract}
Four groups of third grade children were presented with two consecutive two-choice discrimination problems or two double-stimulus oddity problems. One half of the children received these training problems in the visual modality, while the remainder experienced the problems by active touch. All children were then presented with two double-stimulus oddity problems in the alternate modality. During original learning, the tactual oddity task was more difficult than both the visual oddity problem and the tactual discrimination problem. All oddity trained groups learned the crossmodal oddity task at a rate superior to the children given discrimination training; thus, it was concluded that crossmodal transfer of the oddity concept did occur.
\end{abstract}

Blank and Bridger (1964) discussed the distinction between crossmodal equivalence (CME), which involves recognition of a particular stimulus element presented in two distinct sensory modalities, and crossmodal concepts (CMC), which require the utilization of a concept to solve problems involving dissimilar yet analogous stimuli presented in distinct sensory modes.

Smith and Tunick (1969), employing the miniature experiment design (House \& Zeaman, 1963), demonstrated that retarded children possessed the ability to transfer information concerning specific stimulus cues between tactual and visual discrimination problems. However, they were unable to demonstrate any dimensional transfer between these two modalities. Thus, if a child experienced form discrimination in the original learning, his performance on the crossmodal transfer task was facilitated only if the same positive and negative forms were presented, but if two new forms were presented, performance was not facilitated. Similar cue transfer between tactual and visual problems has been demonstrated in normal children by Eastman (1967) and Blank and Altman (1968). These investigators also demonstrated the retardation of learning in the crossmodal transfer task if a reversal of the positive and negative cues occurred between the two problems. Similar cue-specific transfer, lack of dimensional transfer, and crossmodal reversal shift effects have been demonstrated in monkeys (Rothblatt \& Wilson, 1968).

Blank and Klig (1970), using nursery school children, and Tyrrell (1970), with first graders, have demonstrated crossmodal discrimination transfer which is not cue specific. Both of these studies demonstrated intradimensional and extradimensional shift effects between successive tactual and visual problems. These shift effects demonstrate that young children are able to

*The research reported herein was partially supported by Public Health Service Grant 1-R03-MH20332-01 and in part by funds from the Franklin and Marshall Committee on Grants. The author wishes to thank Willard Funk, Principal of Wharton and Ross Elementary Schools, and his staff for their cooperation in the conduct of this investigation. Special recognition is extended to Sherri Stamm for her assistance in data collection.

tRequests for reprints should be addressed to Donald $J$. Tyrrell, Department of Psychology, Franklin and Marshall College, Lancaster, Pennsylvania 17604. transfer information regarding stimulus dimensions of form and texture between two separate modalities and respond in the new modality to the stimulus dimension which was relevant in the original modality even though all new cues of both dimensions were introduced. Thus, if superordinate stimulus properties (dimensions) common to two separate modalities are responded to in one modality, this information can be utilized in either modality.

The present research is an attempt to compare the speed with which third grade children acquire the oddity concept in the haptic and visual modalities and to assess the extent to which they can transfer that concept to problems presented in the alternate modality.

\section{METHOD}

\section{Subjects and Design}

Forty-eight third grade children were randomly assigned to one of four experimental conditions, each of which represented one cell in a 2 (sensory modality) by 2 (problem type) factorial design. All children were presented with a set of two training problems via either the visual (V) or tactual (T) modes of sensory input. One half of the children in each modality condition were given training on a set of two-choice discrimination (D) problems and the remainder a set of double-stimulus oddity (0) problems. Upon completion of this initial set of two problems, all children were presented with two sets of double-stimulus oddity problems in the alternative sense modality (see Table 1). All groups had $12 \mathrm{Ss}$ with the exception of the $T_{0}-V_{0}$ condition, in which one $S$ had to be discarded for failing to attain the learning criterion on the original problem.

\begin{abstract}
Apparatus
A portable version of a modification of the WGTA was the major apparatus. A $60 \times 100 \mathrm{~cm}$ plywood screen was interposed between $E$ and $S$. For visual presentation of the stimulus objects, a stimulus tray could be slid from $E$ to $S$. The tray had two $7-\mathrm{cm}$ circular foam-lined reward wells spaced $45 \mathrm{~cm}$ apart. While the tray was on the $E$ side of the panel not visible to $S$, the stimulus patterns were inserted into the slanted Plexiglas holders which could be placed over the reward wells. The tray was then slid to S's side of the panel, and $S$ responded by sliding the stimulus holder of S's choice. If the response was correct, an M \& M candy was to be found in the reward well and $E$ made an appropriate verbal comment (e.g., "That's good," "That's correct," "You're doing very well"). Following an incorrect choice, no $M$ \& $M$ was seen and $E$ made an appropriate verbal comment (e.g., "No," "Not there," "That's wrong").
\end{abstract}


For tactual problems, the apparatus was altered by replacing the sliding tray with a box, the floor of which was identical to the sliding tray. The side of the box facing $S$ had $15-\mathrm{cm}$ cloth-draped holes with elasticized cuffs directly in line with and $7.5 \mathrm{~cm}$ above each reward well. The side of the box facing $E$ was open and allowed $\mathrm{E}$ to place stimulus objects over each reward well. A tactual trial consisted of S placing both hands through the holes and tactually investigating both stimulus objects simultaneously. S could then push aside the chosen object and feel in the reward well for the $M \& M$ while $E$ made an appropriate verbalization.

Two unique oddity problems were created within each modality. Each problem set consisted of all possible pairs of three different forms. Thus, within each set three pairs contained two similar forms (e.g., X, X) while three pairs included two dissimilar (or odd) forms (e.g., X, T). For visual problems, the stimulus pairs were two-dimensional forms cut from black construction paper and were glued to a $12 \times 16 \mathrm{~cm}$ sheet of white cardboard. Tactual stimuli were smooth plastic objects mounted in pairs on $12 \times 16 \mathrm{~cm}$ Masonite bases. The two visual problem sets consisted of a cross, a $\mathrm{T}$, and an $\mathrm{X}$ in one set, with a star, a hexagon, and an elongated diamond in the second. One set of the tactual problems was comprised of a rectangular solid, a cylinder, and a cone, while the second included a sphere, a pyramid, and a cube. The order of problem set presentation was counterbalanced within each condition so that each set appeared equally often as the first and second problem.

Each trial of an oddity problem consisted of the presentation of two stimulus pairs: one pair of similar forms and one pair of odd forms, with the choice of the latter being reinforced. The pairs to be presented on each trial were randomly selected, and the position of the positive pair was determined by a portion of the Gellermann (1933) series.

Each stimulus used for the discrimination problems consisted of a single form mounted on backgrounds identical to those of the oddity stimuli. Two different discrimination problems were constructed within each modality. The visual problems were both yellow-green discrimination, while the tactual problems were rough-smooth texture problems. Each child in the discrimination training groups experienced a sequence of two problems with the same positive color (or texture). The problems differed in the forms used; the visual problems had either two hexagon or two " $T$ " shapes, while one tactual problem employed two cubes and the other two pyramids. The order of problem presentation and the positive and negative color (or texture) was counterbalanced within each group.

\section{Procedure}

Prior to the data collection, all Ss were randomly assigned to experimental conditions. A problem set sequence was selected for each S, and the positive discrimination cue was determined. Ss were run individually in a small room adjoining the classroom. After the child was shown how the "candy game" worked and was given four practice trials, the experimental trials were initiated and presented at a rate of 25 trials each day until a criterion of 20 correct responses occurred in a single daily session. Each succeeding problem was presented on the day following criterion performance of the previous problem.

Each child was presented two training problems in the training modality followed by two oddity problems in the transfer modality. Two problems were presented to increase the likelihood that the oddity trained Ss were responding on the basis of the oddity-similarity relationships between the elements of the stimulus pairs rather than to a particular cue or set of cues. This latter strategy would produce no transfer between two problems within the same modality and would prevent crossmodal transfer. Thus, performance on the second oddity problem serves as a measure of the extent to which intramodal transfer of conceptual responding has occurred as well as assuring the possibility of crossmodal transfer.

\section{RESULTS}

The primary dependent variable, the mean number of errors to criterion, appears in Table 1 for each of the four groups over the sequence of four problems. A 2 (type of training task) by 2 (training modality) analysis of variance on the errors in the first problem indicated no significant main effects but a significant Task by Modality interaction $(F=9.08 ; d f=1 / 43$; $\mathrm{p}<.005)$. Individual comparisons were performed by Scheffe tests and revealed that significantly more errors were made in the tactual oddity condition than in either the visual oddity or the tactual discrimination conditions. Further Scheffé tests indicated that the difference in errors between the two visual tasks was not significant nor was the difference between the two discrimination problems.

A similar analysis of errors during the second training problem revealed no significant between-group differences. However, the relatively low error scores on this problem for all groups indicates a high level of interproblem transfer. This is particularly meaningful for the oddity groups since interproblem transfer would indicate some conceptual transfer rather than transfer based upon specific stimulus elements.

The most important data relevant to crossmodal transfer are the error rates during the first crossmodal transfer task (Problem 3). A 2 (type of training task) by 2 (transfer modality) analysis of variance on these data indicated that Ss who had received oddity pretraining made significantly fewer errors than those who received discrimination training $(F=9.64 ; d f=1 / 43 ; p<.005)$. No other comparison approached significance.

Another measure of the extent to which oddity training may affect a crossmodal oddity task is the comparison of the errors on the crossmodal oddity task (Problem 3) to the errors on the original oddity task (Problem 1). This comparison over problems can be made within each of the oddity groups as well as between different groups which received the oddity tasks within the same modality on the training and the transfer task. The within-group performance change was assessed by $t$ tests for related means and demonstrated that fewer errors were made on Problem 3 than on Problem 1 for both the tactual to visual group $(\mathrm{t}=5.57$; $\mathrm{df}=10 ; \mathrm{p}<.001)$ and for the visual to tactual group $(t=2.66 ; d f=11 ; p<.05)$. Performance differences between original training and crossmodal transfer between the two groups experiencing training and transfer within the same modality were assessed by $t$ tests for independent means and demonstrated that fewer errors were made during the transfer task in both the visual modality $(\mathrm{t}=4.69 ; \mathrm{df}=21 ; \mathrm{p}<.001)$ and the tactual modality $(\mathrm{t}=5.45 ; \mathrm{df}=21 ; \mathrm{p}<.001)$.

One measure of generalized interproblem improvement in performance which may not be attributable to transfer of the oddity concept is derived 
from a comparison of performance on the first oddity problem (Problem 3) for the Ss who received discrimination training relative to performance on the first oddity problem (Problem 1) for those Ss who received oddity training within each of the modalities. This comparison demonstrated that performance in a tactual oddity task following visual discrimination training and performance in a visual oddity task following tactual discrimination training are not significantly different from performance in an oddity task within that modality without previous training $(\mathrm{t}=1.59, \mathrm{df}=21, \mathrm{p}\rangle .1$ and $\mathrm{t}=\langle 1, \mathrm{df}=22, \mathrm{p}\rangle .5$, respectively).

Finally, performance on the final transfer problem was analyzed by a 2 (type of training task) by 2 (transfer modality) analysis of variance of the errors. This analysis demonstrated that, although fewer errors were made by oddity trained Ss, this difference was only marginally reliable $(\mathrm{F}=3.27 ; \mathrm{df}=1 / 43 ; .10>\mathrm{p}>.05)$.

\section{DISCUSSION}

The analysis of errors to criterion on the initial problem indicated that a tactual oddity problem is more difficult for third grade children than either a discrimination problem in the tactual modality or an oddity problem in the visual modality. This difference in difficulty is not maintained through the transfer problems and is perhaps artifactual, although other investigators (Lobb, 1965; Millar, 1972) have concluded that Ss are less adept at processing information in the tactual modality.

More interesting, however, is the ability of these children to transfer the oddity concept, as a principle of solution, both within and between visual and haptic sensory modalities. Performance on the second training problem for the oddity Ss in both modalities indicated a large decrement in errors which indicates the extent of intramodal transfer.

Crossmodal transfer was assessed by the performance of the children during the third problem, the first transfer problem in the alternate modality. The children who had received oddity training made significantly fewer errors to criterion in this problem than the children who had received the discrimination training. In addition, performance level on this problem was significantly better than the same S's performance on the original oddity problem and better than performance of naive children on an oddity problem in the same modality.

This interproblem performance could be an indication of a more general learning to learn phenomenon rather than an indication of transfer of conceptual responding. Such an explanation would not account for the lack of interproblem performance for children in the discrimination training conditions. These children had previously experienced two learning problems and, presumably, could have acquired the same decrement in irrelevant response tendencies. Yet, they made as many errors on the third problem as naive children did on their first oddity problem.

The level of crossmodal conceptual transfer demonstrated by third grade children within the normal ranges of intelligence and the fact that normal first grade children can transfer dimensional information across sensory modes (Tyrrell, 1970) is discrepant from data obtained from retarded children (Smith \& Tunick,
Table 1

Mean Errors to Criterion for Each Experimental Group Throughout the Series of Four Problems

\begin{tabular}{lccccc}
\hline & \multicolumn{2}{c}{$\begin{array}{c}\text { Training } \\
\text { Problems }\end{array}$} & & \multicolumn{2}{c}{$\begin{array}{c}\text { Crossmodal } \\
\text { Problems }\end{array}$} \\
\cline { 2 - 3 } \cline { 5 - 6 } & 1 & 2 & & 3 & 4 \\
\hline $\begin{array}{c}\text { Discrimination Training } \\
\quad \text { Visual to Tactual }\end{array}$ & 21.3 & .4 & & 32.8 & 6.5 \\
$\quad \begin{array}{l}\text { Tactual to Visual } \\
\text { Oddity Training }\end{array}$ & 19.0 & 1.2 & & 20.3 & 2.7 \\
$\quad$ Visual to Tactual & 28.4 & 1.7 & & 7.1 & .3 \\
$\quad$ Tactual to Visual & 52.4 & 1.3 & & 2.1 & 1.0 \\
\hline
\end{tabular}

1969) and lower primates (Rothblatt \& Wilson, 1968). Further examination of the level of intermodal transfer ability may reveal differences as a function of developmental level. Such a conclusion would also be consistent with the data which demonstrate deficiencies in intermodal attention shifting in retarded readers (Katz \& Deutsch, 1963; Katz \& Deutsch, 1964) and with the speculations of Jensen (1969) regarding crossmodal transfer as a relatively pure measure of Level II (conceptual) ability.

\section{REFERENCES}

Birch, H. G., \& Lefford, A. Intersensory development in children. Monographs of the Society for Research in Child Development, 1963, 28, (Serial No. 89).

Blank, M., \& Altman, L. D. Effect of stimulus modality and task complexity on discrimination and reversal learning in preschool children. Journal of Experimental Child Psychology, 1968, 6, 598-606.

Blank, M., \& Bridger, W. Cross modal transfer in nursery school children. Journal of Comparative \& Physiological Psychology, 1964, 58, 277-282.

Blank, M., \& Klig, S. Dimensional learning across sensory modalities in nursery school children. Journal of Experimental Child Psychology, 1970, 9, 166-173.

Eastman, R. The relative cross modal transfer of a form discrimination. Psychonomic Science, 1967, 9, 197-198.

Gellermann, L. Chance orders of alternating stimuli in visual discrimination experiments. Journal of Genetic Psychology, $1933,42,206-208$

House, B. J., \& Zeaman, D. Miniature experiments in the discrimination learning of retardates. In L. P. Lipsitt and C. C. Spiker (Eds.), Advances in child development and behavior. Vol. I. New York: Academic Press, 1963. Pp. 313-385.

Jensen, A. R. How much can we boost IQ and scholastic achievement? Harvard Educational Review, 1969, 33, 1-123.

Katz, P. A., \& Deutsch, M. Relation of auditory-visual shifting to reading achievement. Perceptual \& Motor Skills, 1963, 17, 327-332.

Katz, P. A., \& Deutsch, M. Modality of stimulus presentation in serial learning for retarded and normal readers. Perceptual \& Motor Skills, 1964, 19, 627-633.

Lobb, H. Vision "versus" touch in form discrimination. Canadian Journal of Psychology, 1965, 19, 175-187.

Millar, S. Effects of interpolated tasks on latency and accuracy of intramodal and cross-modal shape recognition by children. Journal of Experimental Psychology, 1972, 96, 170-175.

Rothblatt, L., \& Wilson, W. A. Intradimensional and extradimensional shifts in the monkey within and across sensory modalities. Journal of Comparative \& Physiological Psychology, 1968, 66, 539-543.

Smith, J., \& Tunick, J. Cross-modal transfer of a discrimination by retarded children. Journal of Experimental Child Psychology, 1969, 7, 274-281.

Tyrrell, D. J. Intradimensional and extradimensional shifts and cross modal transfer in children. Paper presented at Eastern Psychological Association, Atlantic City, 1970.

(Received for publication June 22, 1974.) 